EPJ Web of Conferences 37, 04001 (2012)

DOI: $10.1051 /$ epjconf/20123704001

(C) Owned by the authors, published by EDP Sciences, 2012

\title{
Spectral-statistics analysis of the light meson spectrum
}

\author{
C. Fernández-Ramírez ${ }^{1, a}$, L. Muñoz ${ }^{1,2}$, A. Relaño ${ }^{3}$, and J. Retamosa ${ }^{1}$
}

1 Grupo de Física Nuclear, Departamento de Física Atómica, Molecular y Nuclear, Facultad de Ciencias Físicas, Universidad Complutense de Madrid, Avda. Complutense s/n, E-28040 Madrid, Spain

2 European Centre for Theoretical Studies in Nuclear Physics and Related Areas (ECT*), Villa Tambosi, Strada delle Tabarelle 286, Villazzano (TN), I-38123, Italy

3 Departamento de Física Aplicada I, Facultad de Ciencias Físicas, Universidad Complutense de Madrid, Avda. Complutense s/n, E-28040 Madrid, Spain

\begin{abstract}
Quantum chaos is currently a well established discipline with outreach to many fields of physics. The most important signature of quantum chaos is the statistical analysis of the energy spectrum, which distinguishes between systems with integrable and chaotic classical analogues. The spectral statistical techniques inherited from quantum chaos have been applied to the baryon spectrum revealing its likely chaotic behavior in the low energy regime $[1,2]$. We present a robust analysis of the spectral fluctuations exhibited by the light meson spectrum. With this analysis we can obtain information about the degree of chaos in the spectrum getting insight on the properties of the underlying interactions. Our analysis unveils that the statistical properties of the light meson spectrum are close, but not exactly equal, to those of chaotic systems [3]. Besides the experimental spectrum, we have analyzed several theoretical spectra [4-7] including the latest lattice QCD calculation [8] finding out that, with the single exception of [5], their statistical properties are close to those of a generic integrable system, and thus incompatible with the experimental result.
\end{abstract}

\section{Introduction}

Since Wigner discovered that the statistical properties of complex nuclear spectra are well described by the Gaussian Orthogonal Ensemble (GOE) of Random Matrix Theory [9], spectral-statistics techniques have developed a lot, becoming a very powerful statistical method to study the energy spectra of quantum systems $[10,11]$. One of the most important results in this field is that the dynamical regime of a quantum system can be determined just by analyzing the statistical properties of the energy-level fluctuations, classifying systems in: (i) chaotic; (ii) integrable, or (iii) intermediate. In addition, the energy-level fluctuations of integrable and chaotic systems are universal and integrable systems display a non-correlated sequence of levels, which follows the Poisson distribution [12], while chaotic systems follow the correlation structure described by GOE [13]. Mesons can be considered as aggregates of quarks and gluons with baryon number equal to zero. The mass spectrum of low-lying mesons can be understood as the energy spectrum of a quantum system, like an atomic nucleus, and it consists on all the possible states which stem from an interacting quantum system. Henceforth, the meson spectrum can be studied from the statistical point of view employing spectral-statistics techniques and getting insight on the dynamical regime of light mesons. The spectral-statistics analysis has been already applied to the hadron mass spectrum in [1] obtaining a chaotic-like behavior for both mesons and baryons. In [2] the spectral-statistic techniques were used to compare the experimental baryon spectrum with quark models, focusing on the problem of missing resonances obtaining that

\footnotetext{
a e-mail: cefera@gmail.com
} 
quark models give rise to integrable spectra, while the experimental one is chaotic. Besides, matching theoretical spectra to the experimental one reported by the Review of Particle Physics (RPP) [14] is a tricky business prone to mismatching. Three are the main reasons: (i) The experimental spectrum has missing states, (ii) the theoretical spectra can easily overpredict states, and (iii) the theoretical spectra of quark models do not incorporate exotics or hybrids, so they have missing levels. Spectral-statistics techniques overcome these difficulties in the sense that overall properties of the spectrum are tested instead of level by level matching. If missing levels happen, we understand how this affects the results and a reliable interpretation can be given. Hence, theoretical models must reproduce the spectral fluctuations of the experimental spectra, since they determine the dynamical regime and the complexity of the real interactions. Once this is done, the next step is to match level by level the experimental spectrum.

\section{Statistical Analysis}

Prior to any statistical analysis of the spectral fluctuations one has to accomplish some preliminary tasks. First of all, it is necessary to take into account all the symmetries that properly characterize the system. Mixing different symmetries deflects the statistical properties towards the Poisson distribution $[9,11,15]$. Thus, the spectrum is separated into sequences of energy levels involving the same symmetries, splitting it into sequences of fixed spin $(J)$, isospin $(I)$, parity $(P)$, and $C$-parity $(C)$. Our analysis entails $d=126$ energy levels from the RPP experimental spectrum up to $2.5 \mathrm{GeV}$ of energy. The second task is the unfolding. The energy spectrum of a quantum system is characterized by the level density $\rho(E)$, which can be split into a smooth part $\bar{\rho}(E)$, giving the secular behavior with the energy, and a fluctuating part $\widetilde{\rho}(E)$, which is responsible for the statistical properties of the spectrum. The unfolding consists on removing the smooth component $\bar{\rho}(E)$ of the level density $\rho(E)$. Once the $\widetilde{\rho}(E)$ has been isolated the fluctuations from different systems or from different parts of a single spectrum can be compared. Because the experimental meson spectrum has been divided in very short sequences of levels, we have to use the local unfolding procedure [16] which considers that the variation of $\bar{\rho}(E)$ along a short sequence of energy levels must be negligible, which is a reasonable assumption in this case [1]. The procedure is the following. Let $\left\{E_{i}, i=1,2, \ldots, l_{x}\right\}_{X}$ be an energy-level sequence characterized by the set $X$ of good quantum numbers. Then, the distances between consecutive levels, $S_{i}=E_{i+1}-E_{i}$, are rescaled using their average value $\langle S\rangle=\left(l_{x}-1\right)^{-1} \sum_{i=1}^{l_{x}-1} S_{i}$ to obtain the quantities $s_{i}=S_{i} /\langle S\rangle$, called generically nearest neighbor spacings (NNS). The statistical properties of the NNS are studied through the nearest neighbor spacing distribution (NNSD) [17], denoted $P(s)$. The NNSD follows the Poisson distribution $P_{p}(s)=\exp (-s)$ for generic integrable systems [12] and follows the Wigner surmise $P_{W}(s)=\frac{\pi s}{2} \exp \left(-\frac{\pi s^{2}}{4}\right)$ [13] for chaotic systems with time reversal and rotational invariance. Due to the low statistics it is misleading to compare the NNSD obtained from the experimental data and theoretical models directly to the Poisson distribution, the Wigner surmise or the Berry-Robnik distribution (an intermediate distribution between Poisson and Wigner which is defined as $(1-f) P_{p}(s)+f P_{W}(s)$ where $f$ represents the fraction of chaoticity). This is overcome by building so-called distorted Poisson (DP), Wigner (DW) and Berry-Robnik (DBR) distributions. The distorted distributions are built from the original Poisson, Wigner and Berry-Robnik distributions modifying them to take into account the effects due to the small amount of data, the short length of the sequences, the amount of sequences, and the unfolding. Once the distorted distributions are built they become the right theory to compare with. The details on how the distorted distributions are built and the associated analysis is done can be found in $[2,3,18]$.

\section{Results}

Figure 1 displays the $P(s)$ distribution for the experimental meson spectrum provided by the RPP compared to the DP distribution, the DW distribution and the DBR with $f=0.78$. The value $f$ has been set fitting the DBR to the experimental $P(s)$. We find that the statistical properties of the experimental 


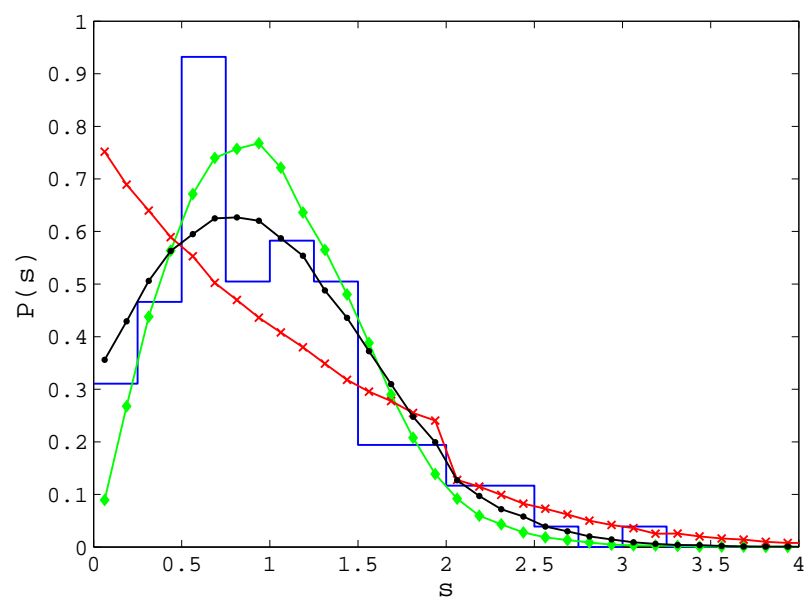

Fig. 1. NNSD for the experimental spectrum (histogram) from the RPP; Distorted Wigner distribution (green line and diamonds); Distorted Poisson (red line and crosses); and Distorted Berry-Robnik with $f=0.78$ (black line and dots).

spectrum are in between Poisson and Wigner statistics. Additionally, one can run a KolmogorovSmirnov hypothesis test where the null hypothesis is that the experimental $P(s)$ coincides with the reference distribution (DP, DW and DBR). The results for the $p$-value are in each case $p_{D P}=0.13$ $p_{D W}=0.47 p_{D B R}=0.68$. Once studied the experimental spectrum one can perform the same analysis for the theoretical spectra available in the literature, obtain their spectral-statistics properties and check if they agree with those of the experimental spectrum. We have done it for the classic model by Godfrey and Isgur [7], the quark models by Koll et al. [4], Vijande et al. [5], Ebert et al. [6], and the latest lattice QCD calculation by the Hadron Spectrum Collaboration (HSC) at $m_{\pi}=396 \mathrm{MeV}[8,19,20]$. We have found that only the quark model by Vijande et al. has a $P(s)$ distribution that agrees with the dynamical regime of the experimental spectrum. The lattice QCD computation as well as the quark models by Ebert et al. and Koll et al. agree with a Poisson distribution. The Godfrey and Isgur quark model is neither Poisson nor Wigner and does not match the experimental properties. Figure 2 displays the $P(s)$ distributions for the quark model from [5] (left) and lattice QCD (right) [8,19,20]. If we fit to a DBR the $P(s)$ obtained from Vijande et al. we obtain $f=0.63$ which is not far from experiment. The lattice QCD result is not surprising because the spectrum is calculated at the unphysical pion mass of $396 \mathrm{MeV}$ and, as the authors acknowledge, further refinements are necessary in order to have a reliable spectrum $[8,19,20]$. The lack of agreement with experiment of most of theoretical models calls for further research on the quark-interaction and the confinement potential. Also, it is necessary a detailed analysis of the model in [5] in order to understand how chaotic behavior can emerge from the quark-antiquark interaction and use that information to refine current models.

The authors thank J.J. Dudek for making available the HSC lattice QCD meson spectrum in [8], and F. Fernández and J. Segovia for providing an updated spectrum of the model in [5]. This research has been conducted with support of the Spanish Ministry of Science and Innovation grant FIS2009-07277 and by CPAN, CSPD-200700042@Ingenio2010. C.F.-R. is supported by "Juan de la Cierva" program of the Spanish Ministry of Science and Innovation (Spain). C.F-R. gratefully acknowledges the Institute of Physics of Jagiellonian University for their hospitality during the conference.

\section{References}

1. V. Pascalutsa, Eur. Phys. J. A 16, 149 (2003) 

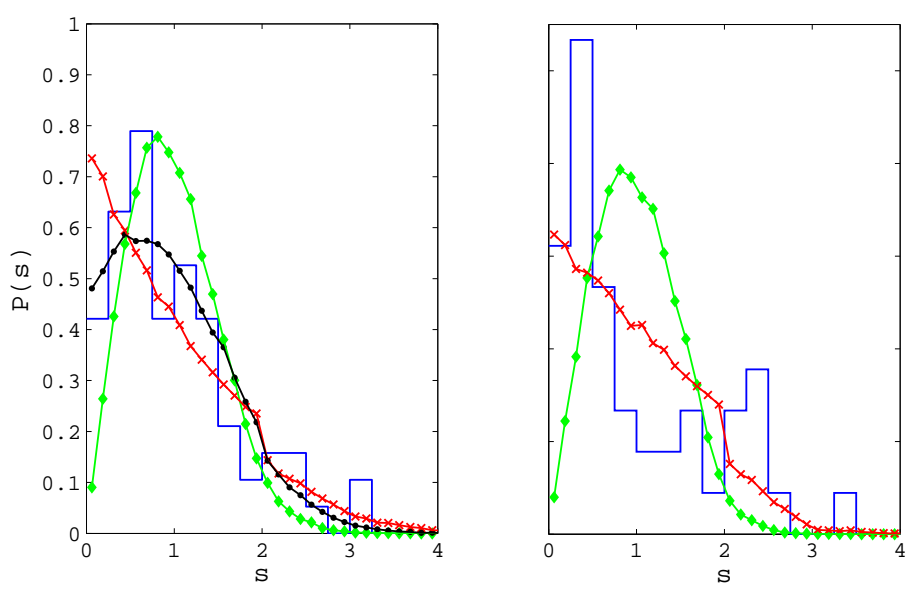

Fig. 2. NNSD for the Vijande et al. [5] quark model (left) and lattice QCD calculation at $m_{\pi}=396 \mathrm{MeV}$ by the HSC (right) from $[8,19,20]$. On both figures the distorted Wigner distribution (green line and diamonds) and the distorted Poisson (red line and crosses) are displayed. In the left figure the Distorted Berry-Robnik with $f=0.63$ is also shown (black line and dots). Similar plots for the other quark models referenced in this proceeding can be found in [3].

2. C. Fernández-Ramírez, A. Relaño, Phys. Rev. Lett. 98, 062001 (2007)

3. L. Muñoz, C. Fernández-Ramírez, A. Relaño, J. Retamosa, Phys. Lett. B 710, 139 (2012)

4. M. Koll, R. Ricken, D. Merten, B. C. Metsch, H. R. Petry, Eur. Phys. J. A 9, 73 (2000)

5. J. Vijande, F. Fernández, A. Valcarce, J. Phys. G 31, 481 (2005); F. Fernández and J. Segovia, private communication (2011)

6. D. Ebert, R. N. Faustov, V. O. Galkin, Phys. Rev. D 79, 114029 (2009)

7. S. Godfrey, N. Isgur, Phys. Rev. D 32, 189 (1985)

8. J. J. Dudek, R. G. Edwards, B. Joó, M. J. Peardon, D. G. Richards, C. E. Thomas, Phys. Rev. D 83, $111502(2011)$

9. C. E. Porter, Statistical Theories of Spectra: Fluctuations (Academic Press, New York, 1965).

10. J. M. G. Gómez, K. Kar, V. K. B. Kota, R. A. Molina, A. Relaño, J. Retamosa, Phys. Rep. 499, $103(2011)$

11. T. Guhr, A. Müller-Groeling, H. A. Weidenmüller, Phys. Rep. 299, 189 (1998)

12. M. V. Berry, M. Tabor, Proc. R. Soc. London A 356, 375 (1977)

13. O. Bohigas, M. J. Giannoni, C. Schmit, Phys. Rev. Lett. 52, 1 (1984)

14. K. Nakamura et al.. (Particle Data Group), J. Phys. G 37, 075021 (2010), and 2011 partial update for the 2012 edition

15. R. A. Molina, J. Retamosa, L. Muñoz, A. Relaño, E. Faleiro, Phys. Lett. B 644, 25 (2007)

16. M. S. Bae, T. Otsuka, T. Mizusaki, N. Fukunishi, Phys. Rev. Lett. 69, 2349 (1992)

17. M. L. Mehta, Random Matrices (Academic Press, New York, 2004)

18. L. Muñoz, C. Fernández-Ramírez, A. Relaño, J. Retamosa, J. Phys.: Conf. Series, in press (2012)

19. J. J. Dudek, R. G. Edwards, M. J. Peardon, D. G. Richards, C. E. Thomas, Phys. Rev. Lett. 103, 262001 (2009)

20. J. J. Dudek, R. G. Edwards, M. J. Peardon, D. G. Richards, C. E. Thomas, Phys. Rev. D 82, 034508 (2010) 